\title{
Piezoelectricity Revealed in the Copolymer of Vinylidene Cyanide and Vinyl Acetate
}

\author{
Seizo MiYata, Masato Yoshikawa, Shigeru TAsaKa, \\ and Munam $\mathrm{KO}^{*}$ \\ Tokyo University of Agriculture and Technology, \\ 2-24-16, Nakamachi, Koganei, Tokyo 184, Japan. \\ *Faculty of Science, Korea University, 1-200 Ogawa-cho, \\ Kodaira, Tokyo 187, Japan.
}

(Received April 11, 1980)

\begin{abstract}
The piezoelectric properties of an alternative copolymer of vinylidene cyanide and vinyl acetate were investigated in connection with the results of dynamic modulus, X-ray diffraction, and electrostriction effect. The rolled and polarized copolymer showed piezoelectricity strong enough to be comparable to that in poly(vinylidene fluoride) films treated by the same poling conditions, but the piezoelectric activity prevailed at high temperature exceeding $100^{\circ} \mathrm{C}$. This strong piezoelectricity is considered due to the introduction of large dipole moments such as $\mathrm{C}-\mathrm{CN}$ groups in the molecule and to the orientation of this large dipole moment in the film.

KEY WORDS Piezoelectricity / Copolymer of Vinylidene Cyanide and Vinyl Acetate / Dipole Moment / Dynamic Modulus / Dielectric Constant / Electrostriction Constant /
\end{abstract}

In the recent years, piezo- and pyro-electric polymers have been gaining an increasing amount of attention owing to their possible application as information transducers. Among these polymers, poly(vinylidene fluoride) has shown the strongest piezo- $^{1,2}$ and pyro-electricity. ${ }^{3}$ These strong activities are considered due to the existence of large $\mathrm{C}-\mathrm{F}$ dipole moments in the molecule and to the oriented polar crystal induced by "poling" polymer films in a high dc field. ${ }^{4}$ From this point of view, if it is possible to synthesize a polymer containing large dipole moments in its molecule and to align the dipole to form a polar crystal, the polymer may be expected to exhibit strong piezo- and pyroelectricity. In regard to the large dipole moment of a cyanide group, piezo- and pyro-electricity in polyacrylonitrile and its copolymer have been studied, but the reported magnitude of their coefficients is not large. ${ }^{5}$ This may be attributable to lack of molecular orientation in these polymers.

In this paper, the results of studies on the piezoelectricity in vinylidene cyanide-vinyl acetate copolymer are reported in connection with dynamic mechanical and X-ray investigations. It is shown that the introduction of large dipole moments such as cyanide groups in the form of vinylidene-type in a polymer molecule and the control of molecular orientation in the copolymer are very effective for bringing about strong piezoelectricity.

\section{EXPERIMENTAL}

Poly(vinylidene cyanide) is so unstable that it undergoes scission by atmospheric moisture at room temperature. Therefore, the copolymer of vinylidene cyanide and vinyl acetate was synthesized by radical polymerization. ${ }^{7}$ This reaction was initiated at $45^{\circ} \mathrm{C}$ in benzene by the addition of benzoyl peroxide. On completion of the reaction, the copolymer began to coagulate and precipitate from a benzene solution. After the coagulum was washed with distilled benzene to remove any nonreactive monomer, the copolymer was dissolved in a few percent concentration of dimethylformamide. The solution was then poured gradually into benzene to reprecipitate for purification.

According to Smith $^{6}$ and Gilbert, ${ }^{7}$ the product of the reactivity ratios for two monomers in a 
polymerization is equal to $5.9 \times 10^{-4}$. This magnitude implies that the reaction of vinylidene cyanide and vinyl acetate yields a copolymer with equimolar portions of the two monomers over a very wide range of change in monomer ratios. The strong tendency of vinylidene cyanide to form alternating copolymer should be noted.

Film specimens were cast from a dimethylacetamide solution. In forming a film, the solution was first spread uniformly on a glass plate and then dried in a vacuum oven at $160^{\circ} \mathrm{C}$ for 4 hours. The films thus obtained were rolled to $200 \%$ strain at $25^{\circ} \mathrm{C}$ and annealed at $150^{\circ} \mathrm{C}$ for $30 \mathrm{~min}$.

Gold electrodes were vacuum evaporated onto both surfaces of the film, and electrical leads were attached to the electrodes by conducting epoxy adhesive. Samples were poled under a dc electric field up to $2 \times 10^{5} \mathrm{Vcm}^{-1}$ for $30 \mathrm{~min}$ at $150^{\circ} \mathrm{C}$. When the samples were cooled to room temperature, the electric field was removed.

The piezoelectric, $d_{31}^{\prime}$, elastic, $c^{\prime}$, and dielectric, $\varepsilon^{\prime}$, constants were measured using the apparatus developed by Fukada et al. ${ }^{8}$ The coordinate axes 1 and 3 of $d_{31}^{\prime}$ refer to the stretching direction and the direction perpendicular to the film surface, respectively. These constants were determined at $10 \mathrm{~Hz}$ as a function of temperature using an automatically null-balancing circuit.

The electrostriction constant was obtained by the method proposed by Furukawa et al. ${ }^{9}$

$\mathrm{X}$-ray diffraction patterns were obtained from the wide-angle diffractometer scans, using $\mathrm{Ni}$-filtered $\mathrm{Cu}-\mathrm{K} \alpha$ radiation.

\section{RESULTS AND DISCUSSION}

Although Smith ${ }^{6}$ has reported that a copolymer of vinylidene cyanide and vinyl acetate shows no crystallinity, broad X-ray reflections appear at $2 \theta=15^{\circ}$ and $2 \theta=30^{\circ}$, respectively as shown in Figure 1. It is apparent that the crystals exist in the copolymer of vinylidene cyanide and vinyl acetate, although this crystallinity is very low. Rolled or stretched samples show rather strong diffraction patterns in the equatorial direction, thus indicating the orientation of the crystals.

However, traces of differential scanning calorimetry (DSC) show no melting endotherm up to $250^{\circ} \mathrm{C}$ at which temperature degradation begins to occur. The complex dynamic moduli $c^{\prime}$ and $c^{\prime \prime}$ are plotted against temperature as shown in Figure 2, where two dispersions are observed. One exists in the hightemperature region at about $125^{\circ} \mathrm{C}$ where the dynamic modulus $c^{\prime}$ decreases rapidly. This temperature is considered as the glass-transition temperature of the copolymer. The other is seen at about $-90^{\circ} \mathrm{C}$. From a comparison with the dispersion of poly(vinyl acetate), this loss-modulus peak may be attributable to the side-chain motion of an acetate group. ${ }^{10}$

The piezoelectric-strain constant $d_{31}^{\prime}$, measured at room temperature for rolled films, was $5 \mathrm{pC} \mathrm{N}^{-1}$ which is ten-times larger than that of the original films. The dielectric constant and dynamic modulus, however, did not change. Thus, the effect of molecular orientation of piezoelectricity is very

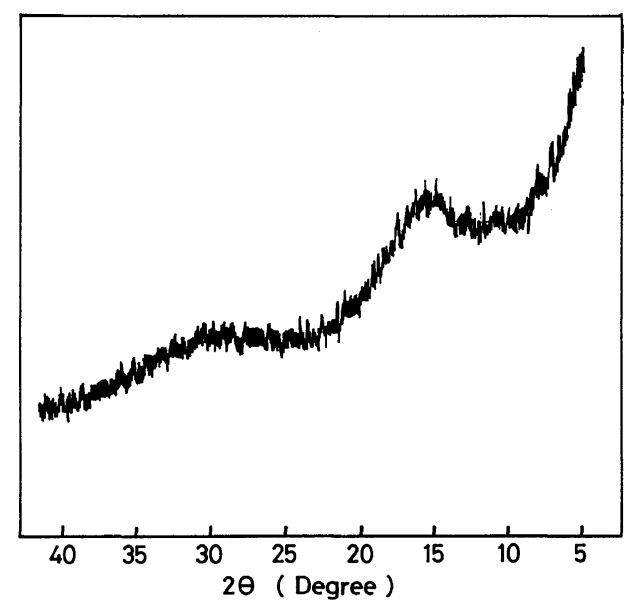

Figure 1. X-ray diffraction scan for copolymer of vinylidene cyanide and vinyl actate.

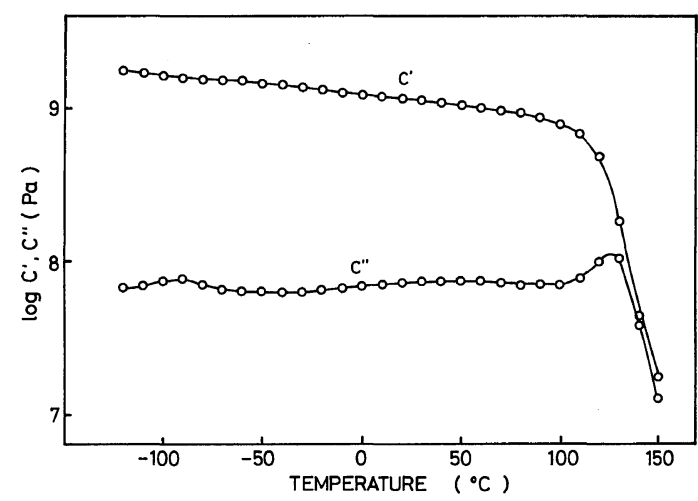

Figure 2. Temperature dependence of elastic constants $c^{\prime}$ and $c^{\prime \prime}(10 \mathrm{~Hz})$. 
remarkable. Figure 3 shows the piezoelectric-strain constant $d_{31}^{\prime}$ for rolled films poled at various poling strengths. The piezoelectric activity increases linearly with poling field. At low polarizing field, the existence of a threshold is observed.

Figure 4 shows the temperature dependence of the $d_{31}^{\prime}$ constant. The two retardational peaks appear at the same dispersion temperatures as those observed in dynamic modulus. The steep increase in the $d_{31}^{\prime}$ constant above $100^{\circ} \mathrm{C}$ results mainly from two effects. One is a decrease in dynamic modulus in

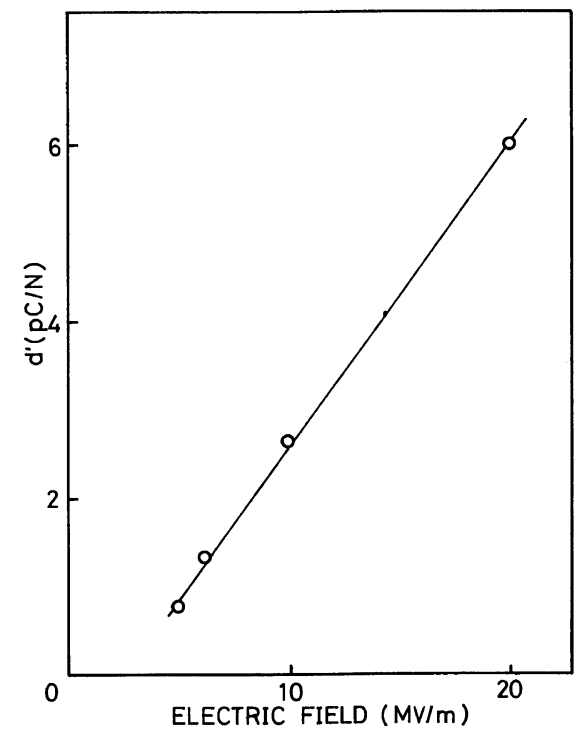

Figure 3. Electric-field dependence of piezoelectricstrain constant $d_{31}^{\prime}(10 \mathrm{~Hz})$.

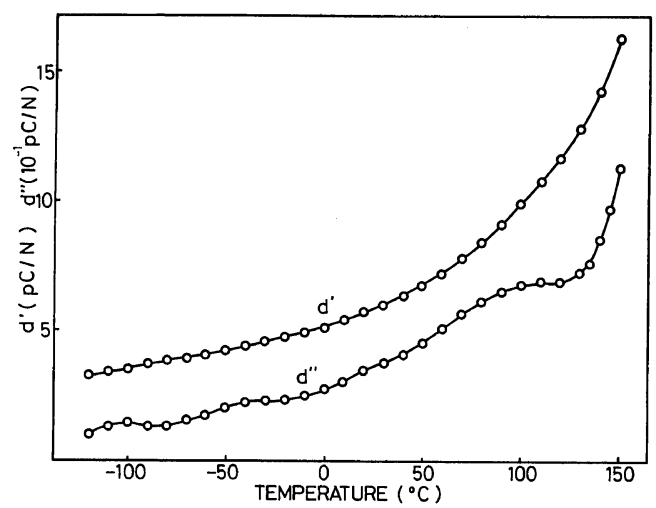

Figure 4. Temperature dependence of piezoelectricstrain constants $d_{31}^{\prime}$ and $d_{31}^{\prime \prime}(10 \mathrm{~Hz})$.

changing from the glassy to rubbery state as shown in Figure 2, the other is due to the increase in the electrostriction constant. ${ }^{11}$

The results are summarized in Table I where a comparison of the data of poly(vinylidene fluoride) (PVDF) and poly ( $\gamma$-benzyl-L-glutamate) (PBLG) is made. Vinylidene cyanide and vinyl acetate copolymer show almost the same piezoelectric activity as that of PVDF in a temperature range from $20^{\circ} \mathrm{C}$ to $100^{\circ} \mathrm{C}$ and four to ten-times larger than that of PBLG. On the other hand, a smaller dielectric constant of the copolymer induces a higher voltage than that observed in PVDF when stress is applied.

There exists a large difference in the physical state between the copolymer and PVDF. For the copolymer, the temperature measured is below its glass-

Table I. A comparison of the data of poly(vinylidene fluoride) and poly( $\gamma$-benzyl L-glutamate) ${ }^{\mathrm{a}}$

\begin{tabular}{|c|c|c|c|c|c|c|}
\hline \multirow{2}{*}{ Material } & \multicolumn{2}{|c|}{$d^{d}$} & \multicolumn{2}{|c|}{$\varepsilon^{e}$} & \multicolumn{2}{|c|}{$\kappa=(\partial \varepsilon / \partial S)^{\mathrm{f}}$} \\
\hline & $20^{\circ} \mathrm{C}$ & $100^{\circ} \mathrm{C}$ & $20^{\circ} \mathrm{C}$ & $100^{\circ} \mathrm{C}$ & $20^{\circ} \mathrm{C}$ & $100^{\circ} \mathrm{C}$ \\
\hline Poly $\left(\right.$ VDCN + VAc) ${ }^{b}$ & 5.0 & 10.0 & 6.0 & 6.0 & -4.3 & -3.0 \\
\hline $\mathrm{PVDF}^{\mathrm{c}}$ & 7.0 & 10.5 & 13.0 & 18.0 & 20.0 & 15.0 \\
\hline PBLG & 1.3 & 1.0 & 3.5 & 4.0 & - & - \\
\hline
\end{tabular}

a $(10 \mathrm{~Hz})$.

b $E_{\mathrm{p}}, 20 \mathrm{MV} \mathrm{m}^{-1} ; T_{\mathrm{p}}, 150^{\circ} \mathrm{C} ; t_{\mathrm{p}}, 1 \mathrm{~h}$.

c $E_{\mathrm{p}}, 20 \mathrm{MV} \mathrm{m}^{-1} ; T_{\mathrm{p}}, 110^{\circ} \mathrm{C} ; t_{\mathrm{p}}, 0.5 \mathrm{~h}$.

d $d$, piezoelectric-strain constant.

e $\varepsilon$, dielectric constant.

f $\kappa$, electrostriction constant. 
transition temperature, but above the glasstransition temperature for PVDF. Piezoelectricity of polymers increases rapidly near their glasstransition temperatures with an increase in electrostriction constants and a decrease in dynamic moduli. If both polymers are compared below the glass-transition temperature, the copolymer exhibits piezoelectricity ten-times higher than that of PVDF.

In conclusion, the introduction of large dipole moments such as $\mathrm{C}-\mathrm{CN}$ groups in the polymer molecule and control of its molecular orientation lead to strong piezoelectricity. This can be done best in polymers other than PVDF.

Acknowledgment. This work was supported in part by a Grant-in-Aid for Scientific Research from the Ministry of Education, Science, and Culture, Japan and by the Aid of the Akai Foundation.

\section{REFERENCES}

1. S. Miyata, S. Tasaka, K. Sakaoku, and Y. Ando, Extended Abstracts, International Workshop on Electric Charges in Dielectrics, Kyoto, Octover 9 12, 1978, p 96.

2. T. Furukawa, T. Goho, M. Date, T. Takamatsu, and E. Fukada, Kobunshi Ronbunshu, 36, 685 (1979).

3. G. Pfister, M. Abkowitz, and R. G. Crystal, J. Appl. Phys., 44, 2064 (1973).

4. H. Kawai, Jpn. J. Appl. Phys., 8, 975 (1969).

5. H. Ree, R. E. Salomon, and M. M. Labes, J. Appl. Phys., 50, 3773 (1979)

6. R. D. Smith, Text. Res. J., 27, 730 (1957).

7. H. Gilbert, J. Am. Chem. Soc., 78, 1669 (1956).

8. T. Furukawa and E. Fukada, J. Polym. Sci. Polym. Ed., 14, 1979 (1976).

9. T. Furukawa, J. Aiba, and E. Fukada, J. Appl. Phys., 50, 3615 (1979).

10. K. Schmieder and K. Wolf, Kolloid Z., 134, 149 (1953).

11. S. Tasaka and S. Miyata, Kobunshi Ronbunshu, 36, 689 (1979). 